\title{
Internet of Things Based Smart Health Monitoring of Industrial Standard Motors
}

\author{
R. Gayathri, Shriram K Vasudevan \\ Department of Computer Science and Engineering, Amrita School of Engineering, India
}

\begin{tabular}{l} 
Article Info \\
\hline Article history: \\
Received Mei 17, 2018 \\
Revised Sep 20, 2018 \\
Accepted Nov 15, 2018 \\
\hline
\end{tabular}

\section{Keyword:}

Adafruit

Failure Analytics

Industrial Automation

MQTT

Predictiive Maintenance

Real-time Monitoring

\begin{abstract}
The Industry 4.0 vision provides recommendations how companies can ease the challenges. In an industrial environment, it is beneficial to have a predictive approach to make smart industry using IoT. The Predictive approach includes automating the maintenance activities of machines which help to deliver safety, performance, customer experience, capacity, cost efficiency and sustainability of the key business assets. It also improves the precision and accuracy of data collection, introducing data analytics, removing human bias, improving reproducibility. This will improve information about asset condition, inform inspection and repair schedules based on asset risks. By implementing predictive and preventive maintenance, one can improve equipment life and avoid any unplanned maintenance activity and thus reducing unscheduled downtime. We in this work have a unit which could be easily attached to the motor units and this does not demand any wiring to carry out. The sensor monitor signals from the motor, accurately measuring key parameters at regular interval of time, as desired. And, the data is sent to the cloud, which in our case is adafruit. From there, the data is analysed and it produces meaningful information. The server then sends alert to the users about critical data of machine which can be used for taking corrective actions).
\end{abstract}

Copyright $\odot 2018$ Institute of Advanced Engineering and Science. All rights reserved.

\section{Corresponding Author:}

Shriram K Vasudevan,

Department of Computer Science and Engineering,

Amrita School of Engineering,

Coimbatore, Amrita Vishwa Vidyapeetham, India.

Email:kv_shriram@cb.amrita.edu

\section{INTRODUCTION}

In industries, system downtime can be detrimental; surprise power outrages can create severe issues. It often requires costly repairs that increases overall operating expenses. The tangible cost of downtime go way beyond the salaries of employees who sit idle while waiting for equipment to get back up and running. If damage to equipment occurs, the costs to repair or replace it need to be factored in as well, along with any specialized technical support. The Smart sensor device can help eliminate unplanned equipment downtime with predictive analytics, and measuring data, such as vibration, temperature and other environmental factors. Software monitors any changes in parameters of a machine's operation and automatically trigger an alert for a user or service technician to repair or replace parts prior to failure or unplanned downtime. Using smart sensor app user can check the status of their motors at any time with their smartphone. User can also receive clear recommendations on how to optimize maintenance and save costs. A Vanson Bourne survey of 450 UK, US and French IT decision makers in field service and service management has estimated that the average cost of downtime is $\$ 260,000$ per hour. Research [1]-[3] reveals the loss of industrialists due to unplanned downtime of machines. Bourassa et al in [4] reveals the equipment failures and their contribution to industrial incidents and accidents in the manufacturing industry. 


\section{LITERATURE SURVEY}

There are some products and papers out in the market with the same tag line as we have proposed. But, the real time monitoring and inclusion of the cloud based analytics makes our proposal stand better and in this section, we present a quick summary of the exisitng products. To make the literature survey crisp, only few papers have been cited.

In this [5] a fever alarm armband (FAA) is used to monitor the temperature of the patient. When the high temperature is detected, it raises sound as an alert. In this paper [6], sensor network technology measures the running status of well fuel equipment and data are sent automatically to control center. From there data are analyzed which ensures the safety of oil pump of each well. Thus it helps to predicts the fault of equipment thereby saving money and time.

There are many other research papers which deals with the automatic street lighting, agriculture based applications, education applications etc [7]-[10]. With IoT, the possibilities are infinite. We have tried to use the same with the industrial motors to monitor the health status

\section{SYSEM ARCHITECTURE}

One can refer to the Figure 1 which is self explanatory and easier to understand the architectural flow. Initially when a motor runs i.e. started, each sensor sends the data sensed / measured to cloud for further processing/storage. From there, data is analyzed real time. It helps to determine the current state of the machine. Also, this helps to monitor the defects of machine to predict equipment maintenance requirements and imminent failures. It enables the user to respond to the problems right away, without delay thereby ensuring minimal time loss and expenditure. To predict equipment failure, it is important that organizations with mission-critical assets will invest in advanced analytics to help ensure safe and reliable operations. All aspects of industrial automation can be beneficial using Machine learning. It helps to monitor and perform maintenance on production machinery.

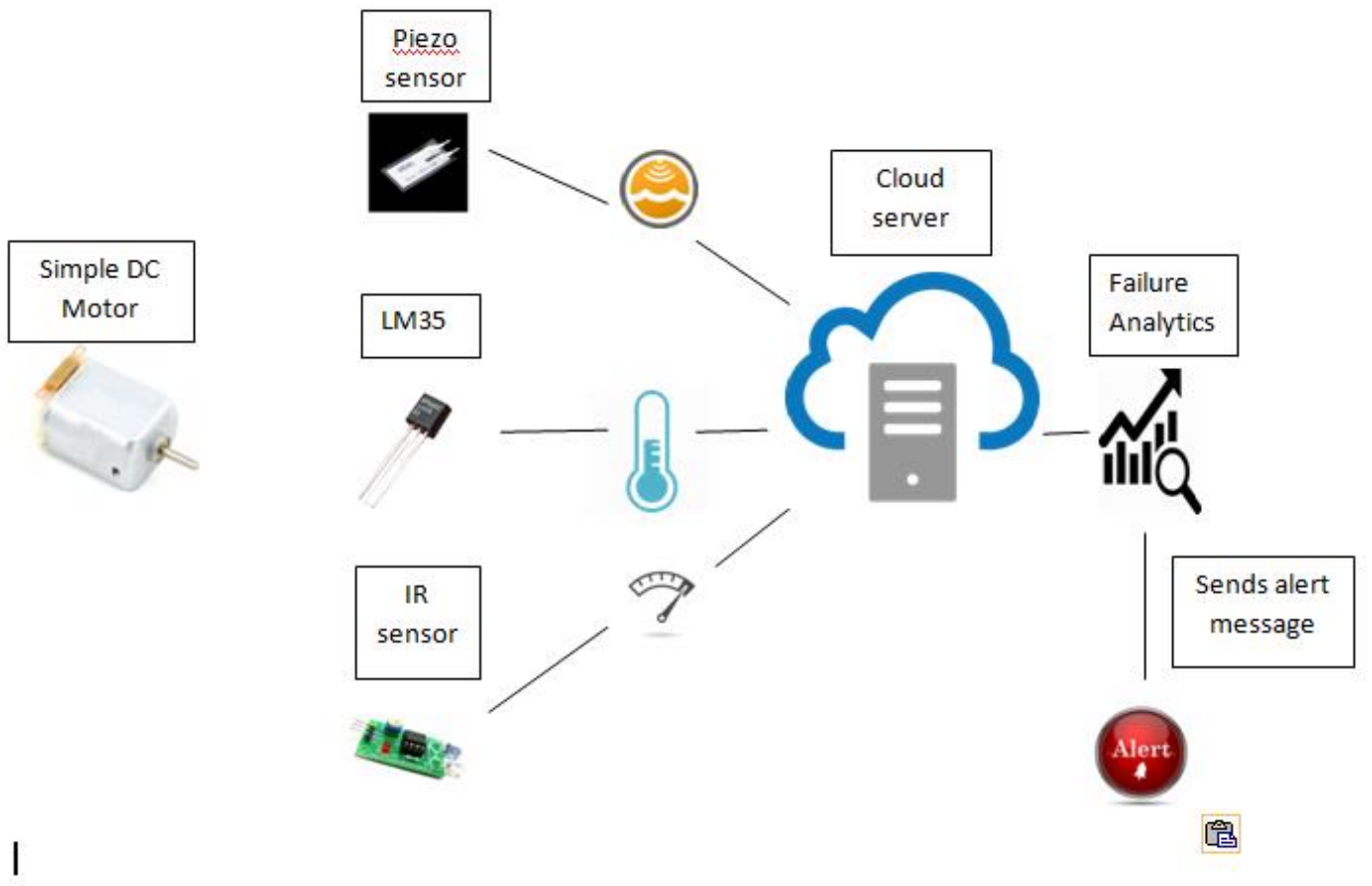

Figure 1. Diagram for overall architecture

\section{SYSTEM FUNCTIONING AND COMPONENTS}

\subsection{Overall vibration}

Vibrations in machines usually occur because of the dynamic effects of manufacturing tolerances, clearances, rolling and rubbing contact between machine parts. They can also be caused by imbalances in the rotating parts, uneven frictions. In practice it is very difficult to avoid vibration in motors. 
Piezo sensors are used for measuring, displaying and analyzing linear velocity, displacement and proximity or acceleration. The Piezo is grounded on one end. The generated voltage is routed to the Arduino's A0 ADC pin.

\section{Working of Piezo sensor:}

Piezo sensor uses the piezoelectric effect that has the ability of to generate an electric charge when mechanical stress is applied. Piezoelectric effect is used to measure changes in acceleration, strain, pressure, and force by converting them into electrical charge.

It causes electric dipole moments of solids due to the pressure applied. Piezo film sensors can be affixed to a motor and the amplitude and frequency of the vibrating structure can be monitored. Using the sensor reading, once the fault and severity are determined, then that machine can recommend a repair and generate a work order.

\subsection{Skin Temperature}

The LM35 sensor is used to measure temperature with an electrical output proportional to the temperature (in degree celsius). LM35 sensor can measure temperature more accurately than using a thermistor. The LM35 IC has 3 pins. 2 pins for the power supply and one for the analog output. Excessive heat causes rapid deterioration of motor. Temperature of motor indicates any abnormality before any serious damage occurs. Hence, it is an important parameter in motor operation.

\subsection{RPM - Rotation per Minute}

Knowing the rotational speed of a device, helps to inspect whether the machinery is running within established parameters or not. The aim of measuring rotation per minute of motor is to measure the losses of an electrical motor and to analyse the influence of load on the losses. If rpm raises than normal, it leads to rise in temperature of engine oil and when they are in contact with the cylinder side walls they move up and reach out the piston rings. Prolonged exposure of very high temperature affects piston rings and they lack sealing the combustion pressure. Thus measuring the rotational speed of a spinning object is important.

The most common way to measure the RPM of a motor is to make a mark on some part of the rotating element which can be sensed with an optical sensor to give an electronic pulse (change of voltage state) once per revolution.

\subsection{Sensor Data Upload to the Cloud}

Sensor is used to detect events or changes in its environment and send the information to other system. Sensor data is like any other data coming from different sources which needs cleansing and analysis. It reports the condition of motor and its performance which enables the users to put intelligence into their maintenance. The sensor monitors signals from the motor. It measures the key parameters at regular intervals and the data is uploaded to cloud.

Adafruit supports MQTT which stands for Message queue telemetry transport. MQTT is a protocol used for device communication that Adafruit I/O supports. Using MQTT library or client, the user can publish and subscribe to a feed to send and receive feed data. Feeds are the basis of the Adafruit IO system. The feed holds metadata about the data that the user push to Adafruit IO. User can push data to Adafruit IO by logging into Adafruit IO and using unique AIO key. The feed also contains the sensor data values which get pushed to Adafruit IO from the user's device. Dashboards allows users to visualize data.

\subsection{Data Analysis}

Sensor data is a stream of information. While the motor is running, it continuously checks the status of key parameters and sends an alarm to the user if it detects a problem. It transmits the abnormal sensor data and sends real-time information on organization's various assets. For each health parameters there will be a preset limits. If the bearing related data exceeds preset limits, an alarm is therefore triggered and sent to the user by SMS text message. This provides an early warning about the maintenance and the user get information about the underlying problem that caused the alarm.

The complete workflow is presented as a diagram in Figure 2. Readers can understand the idea and implementation better from the pictorial representation. 


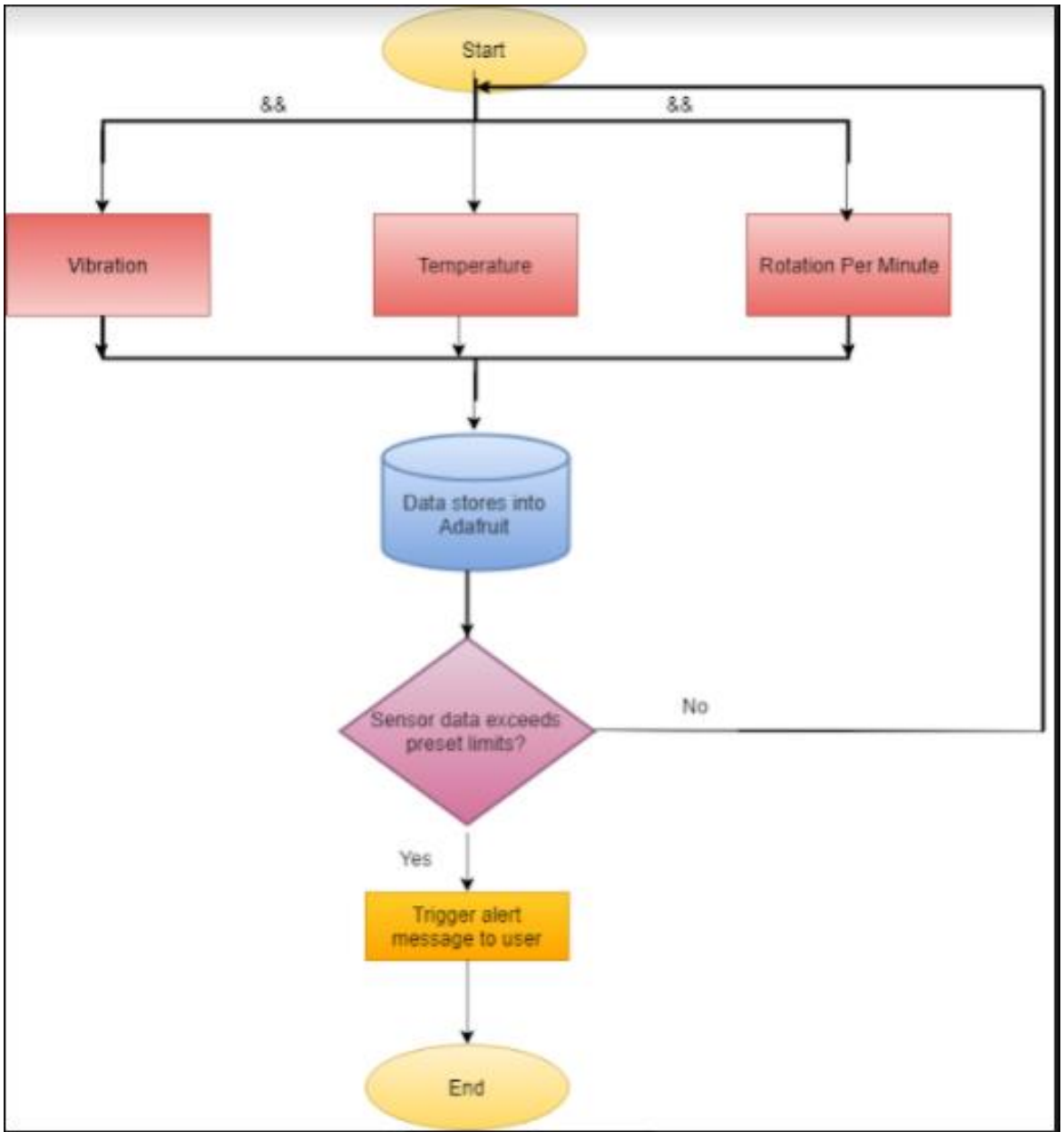

Figure 2. Diagram for work flow

\section{RESULTS AND OBSERVATIONS}

The following sequence of figures shall let the reader visualize the complete workflow and functioning of the proposed product.

After connecting sensor to the motor, and when motor runs, the sensor starts taking its reading and pass the data to cloud. All these data are collected under respective feeds created in the ADAFRUIT. The same is revealed in the Figure 3 Figure 4 represents the realtime vibration data received in the cloud. The fluctuation can be seen in the curve which reveals that, there is vibration in the motor to which our equipment is fit. Figure 5 shall be helpful to visualize the temperature data. The temperature is sensed from the motor skin, realtime. RPM data is presented in the screenshot presented as Figure 6.

\begin{tabular}{|c|c|c|c|c|}
\hline Group / Feed & & Key & Last value & Recorded \\
\hline$\Theta \square$ Default & & default & & \\
\hline 0 rpm & $\theta$ & rpm & 1 & 2 months ago \\
\hline ( Temperature & $\theta$ & temperature & 20 & 2 months ago \\
\hline 0 Vibration & $\theta$ & vibration & 1 & 2 months ago \\
\hline ( Welcome Feed & 0 & welcome-feed & 28 & 2 months ago \\
\hline
\end{tabular}

Figure 3. Feeds from ADAFRUIT 


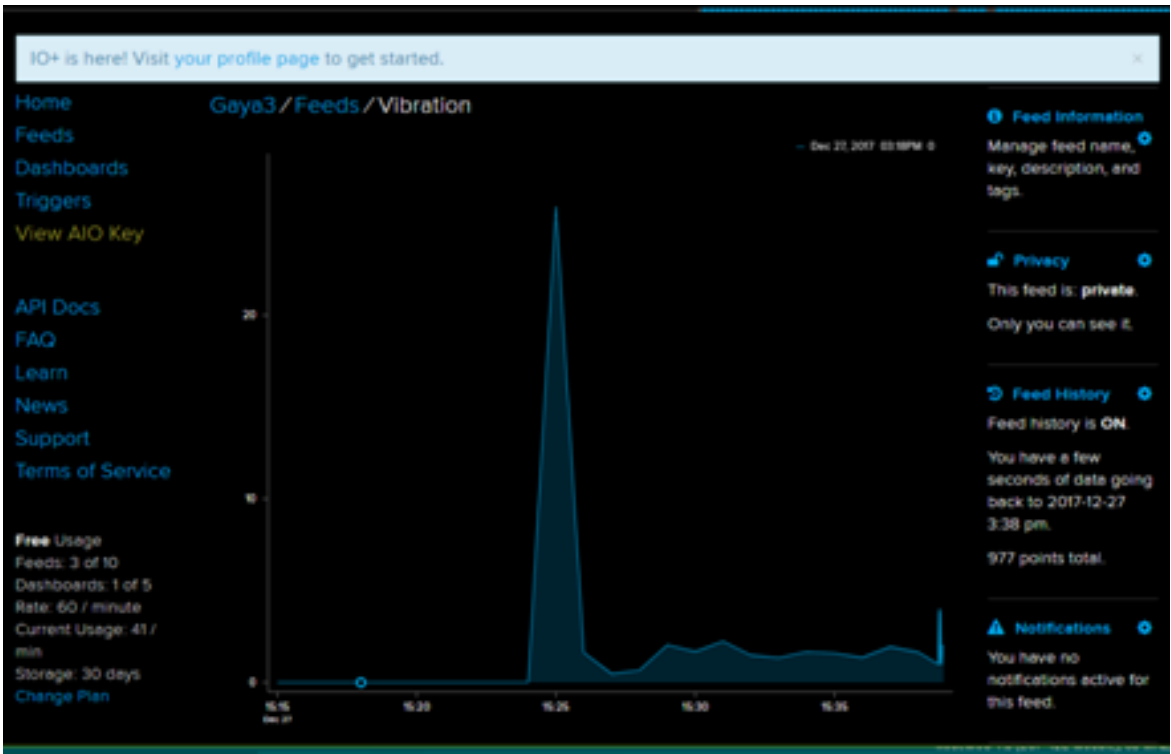

Figure 4. Vibration data received in the cloud

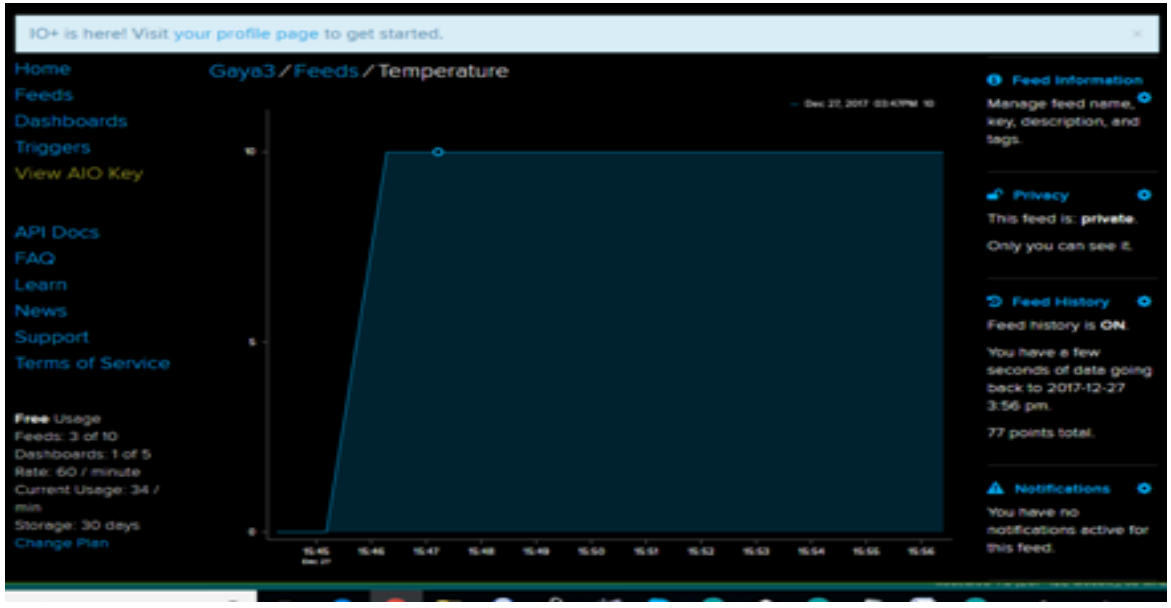

Figure 5. Skin temperature data

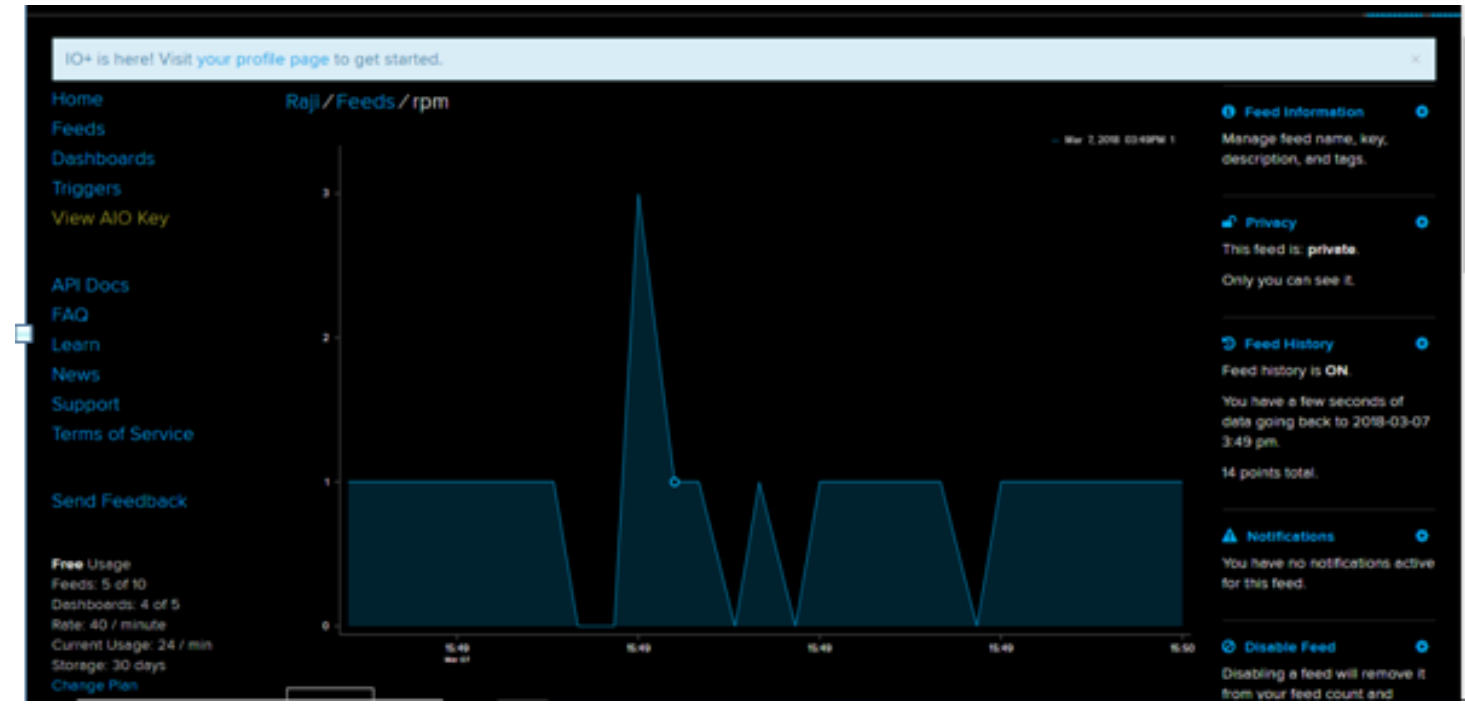

Figure. 6 RPM data 
The above Figure 7 is helpful in understanding how the boundary limits are set for the RPM data with IFTTT. Figure 8 is presenting the boundary limit set details for the vibration data. The appreciable point to note is, all these happen real-time.

The final result is here. When the abnormality is sensed, the alert is triggered immediately as an email. One can see this from Figure 9.

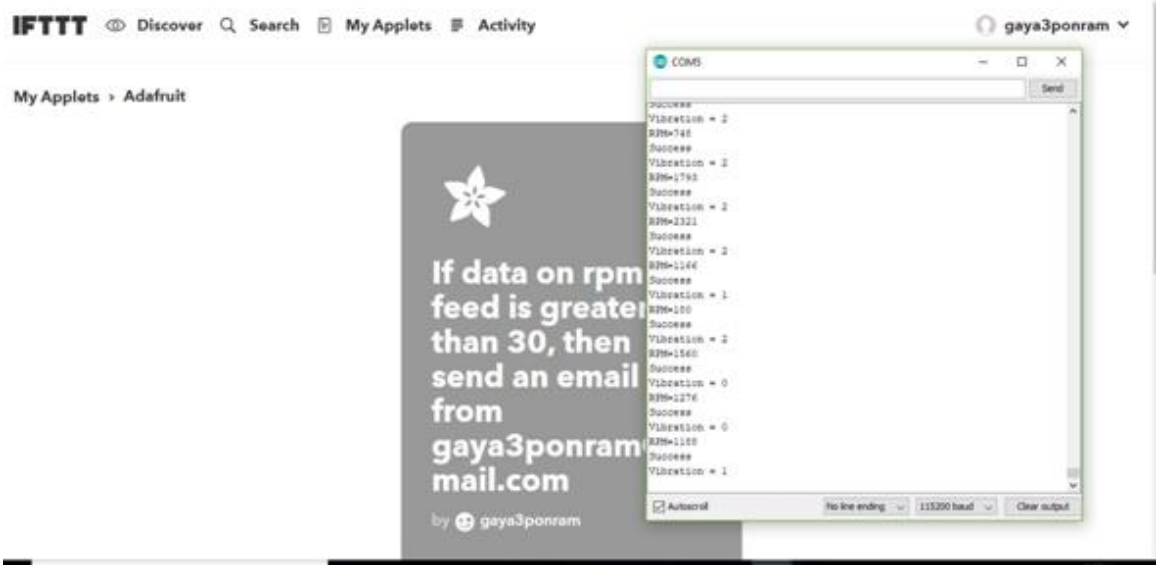

Figure 7. Screenshot for creating IFTT to set boundary limits for rpm data to send alert message

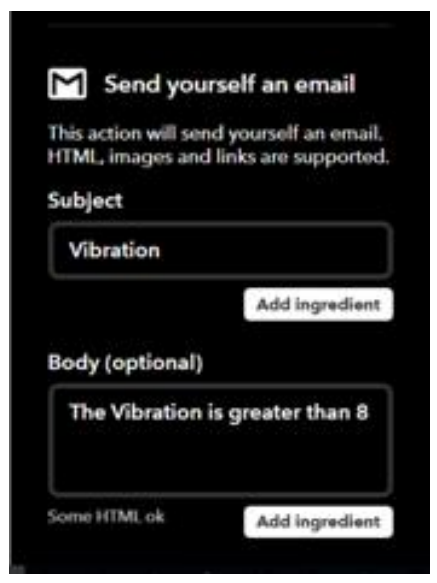

Figure 8. Screenshot for creating IFTT to set boundary limits for vibration data to send alert message

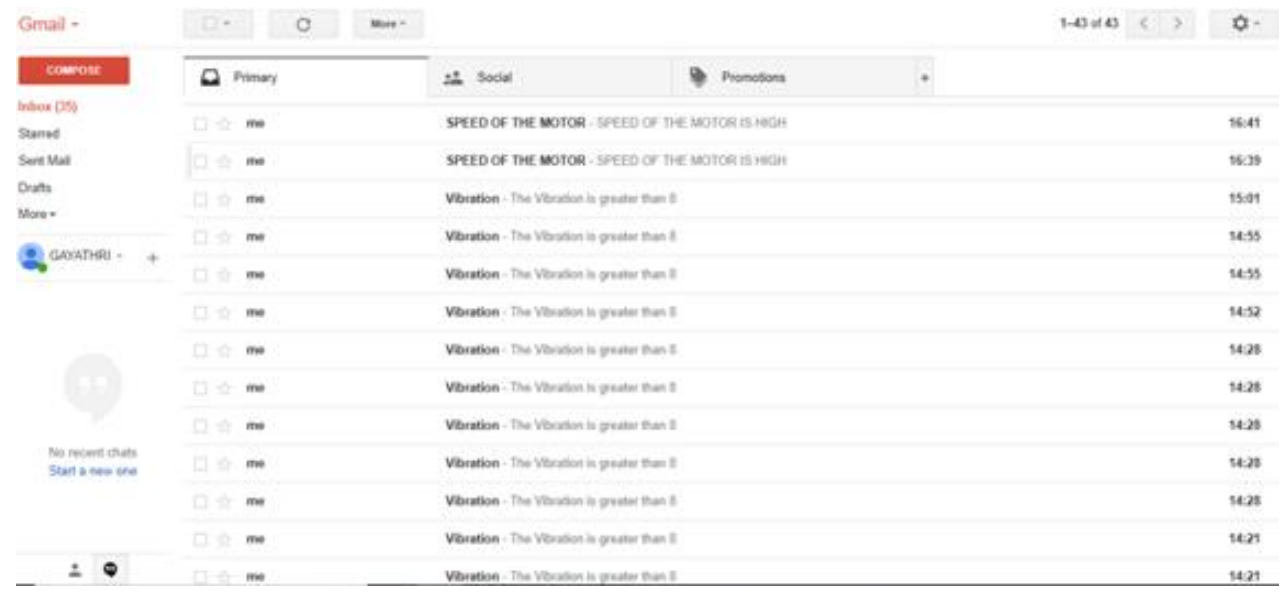

Figure 9. Screen shot for sending alert message through mail 


\section{RESULTS AND OBSERVATIONS}

The way IoT has been growing is tremendous and appreciable [11], [12]. Implementing the predictive maintenance, gives a full view on expenses related to maintenance costs, including forecasts. This prevents unnecessary maintenance procedures and possibility of causing additional problems. The equipment condition is monitored and the component faults are identified, measured and sends alert message to user. This assures machinery reliability. The smart sensor device monitors the key parameters of machine and if there is any change in machines operation, it intimate the user to predict unplanned downtime and equipment failure. By scheduling maintenance activity systematically alleviates workplace pressure on a transparent platform. This technology allows industry, utility and infrastructure customers to make use of data in optimizing their operations. It helps to identify defects of machine at an early stage, while there is still time to take action. In future, we plan to use machine learning as a core component to make system's functioning more independent. Also, we plan to carry out more data analysis related challenges to make this product real meaningful.

\section{REFERENCES}

[1] Cliff Saran, "Millions being lost due to downtime in industrial systems," http://www.computerweekly.com/news/450428532/Millions-being ... systems, Oct 2017.

[2] Tim Ford, "Reducing Unplanned Downtime with Predictive Maintenance," https://www.gepowerconversion.com/inspire/reducing-unplanned-downtime-predictive-maintenance, May 2015.

[3] Mark Stevens, "The Real Cost of Unplanned Downtime-How to Avoid the Risks," https://www.wipfli.com/insights/blogs/manufacturing-tomorrow-blog/170628---the-real-cost-of-unplanneddowntime, Jun 2017.

[4] Bourassa D, Gauthier, Abdul-Nour, "Equipment failures and their contribution to industrial incidents and accidents in the manufacturing industry," https://www.ncbi.nlm.nih.gov/pubmed/26652772

[5] Makoto Takamiya, "Energy Efficient Design and Energy Harvesting for Energy Autonomous Systems," IEEE Conference Publication, Jun 2015.

[6] Shengquan Yang, Ceng Gong, "Research of Petroleum Well Fuel Pump Measurement \& Control System Based on Internet of Things Technology," International Conference on Computer Network, Electronic and Automation, 2017.

[7] Patil, V.C., Al-Gaadi, K.A., Biradar, D.P. and Rangaswamy, M., "Internet of things (Iot) and cloud computing for agriculture: An overview," Agro Informatics and Precision Agriculture (AIPA 2012), 2012.

[8] Parthasarathi, V., Surya, M., Akshay, B., Siva, K.M. and Vasudevan, S.K., "Smart control of traffic signal system using image processing," Indian journal of Science and Technology, vol. 8(16), p. 1, 2015.

[9] Barakat, S., "Education and the Internet of Everything," Int. Bus. Manag, vol. 10(18), pp. 4301-4303, 2016.

[10] Velusamy, K., Venkitaramanan, D., Vasudevan, S.K., Periasamy, P. and Arumugam, B., "Internet of things in cloud," Journal of Engineering and Applied Sciences, vol. 8(9), pp. 304-13, 2013.

[11] Abhishek, S. N., Shriram K. Vasudevan, Prashant R. Nair, S. Thangavelu, and R. M. D. Sundaram, "A Proposal for Mitigating Fishermen Killing In Indian Sea Borders through Technology-Maritime Boundary Identification Device," Indonesian Journal of Electrical Engineering and Computer Science, vol. 6(3), pp. 704-710, 2017.

[12] SriDutta, Rama Ganapathy, Sini Raj, Shriram K Vasudevan, Abhishek SN, "An Inventive and Innovative Alternate for Legacy Chain Pulling System through Internet of Things," Indonesian Journal of Electrical Engineering and Computer Science, pp. 688-694. 